\title{
A High Matrix Tolerance Cadmium Determination Method: An Analysis Method Combining Microextraction and Laser Ablation Inductively Coupled Plasma Mass Spectrometry
}

\author{
Tsing Hai Wang, Yi-Jung Sung, Yi-Kong Hsieh, Chu-Fang Wang* \\ Biomedical Engineering and Environment Sciences, National Tsing Hua University, Taiwan \\ Email: ${ }^{*}$ cfwang@mx.nthu.edu.tw
}

Received December 2013

\begin{abstract}
Herein, we proposed a high matrix tolerance analytical method, using a combination of ammonium pyrrolidine dithiocarbamate/methyl isobutyl ketone (APDC-MIBK) microextraction and laser ablation inductively coupled plasma mass spectrometry (LA-ICP-MS), for Cd concentration determination in aqueous samples. Only $200 \mu \mathrm{L}$ of organic solvent was used throughout the entire analysis process, with enhancement factors as high as 25 . Recoveries from replicate analyses of

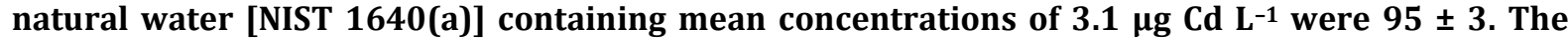
corresponding Cd detection limit was $0.6 \mu \mathrm{g} \mathrm{L}^{-1}$. The main advantage of this approach is its simplicity in terms of sample preparation, as demonstrated by quantifying the Cd levels in tap water, groundwater, and seawater, using a standard addition method.
\end{abstract}

Keywords

Microextraction; LA-ICP-MS; Green Chemistry; APDC; MINEQL

\section{Introduction}

The development of a green analytical route that proceeds with the minimal consumption of chemical reagents but performs with sensitivity as high as possible remains a goal for all analytical chemists. There are two reasonable strategies toward reaching such a challenging objective: one is to upgrade the sensitivity of the analytical instruments and the other is to optimize the sample preparation process. The objective when preparing a sample is to concentrate the analytes of interest to a level sufficiently high to exceed the detection limit of the analytical instrument, while removing any potential interferents; cost-effectiveness and operational convenience are also important issues of concern. Accordingly, microextraction has become one of the most popular sample

${ }^{*}$ Corresponding author. 
preparation methods. Microextraction methods can be divided into two major groups: solid phase microextraction (SPME) (Iraji et al., 2012) and liquid phase microextraction (LPME) (Meeravali et al., 2012; Shaha et al., 2011; Beiraghi et al., 2012; Tehrani et al.; 2010; Zeng et al., 2012). The chemical mechanism of the former involves the partitioning of analytes between liquid and solid phases; in the latter, it is more or less related to chemical equilibrium occurring between aqueous and organic phases.

Despite many successful extraction methods having been reported for analyzing a variety of environmental samples, most of these analytical routes require the consumption of considerable amounts of solvents, both to extract the analytes from the samples and to introduce them into the analytical instruments (Bendicho et al., 2012; Moradi \& Yamini 2012). Although the flame atomic absorption spectrometer (AAS), inductively coupled plasma mass spectrometry (ICP-MS), and inductively coupled plasma (ICP-OES), do not consume any organic solvent during analysis, all of them require certain degree of sample pretreatment to remove the interferences. For example, analytes residing in the high ionic strength are not able to directly introduce into these instruments since clogging can occur as a result of dissolved solids depositing at the interfaces of the components. These salts will not only deteriorate the analytical performance but also greatly increase the need for maintenance.

Surface analysis techniques, which are applied widely in material analysis, appear to be promising analytical routes because they require almost no solvent during the entire analysis procedure. At present, the most relatively accessible techniques for surface analysis include X-ray photoelectron spectroscopy (XPS) (Batrusaitis et al., 2012), scanning electron microscopy combined with energy disperse spectroscopy (SEM/EDS) (Wang et al., 2010) and X-ray fluorescence spectroscopy (XFS) (Howard et al., 2012; West et al., 2011); although these methods are all capable of quantitative analysis of solid samples without the need for organic solvent, their sensitivities are rather low — on the order of a few percentage. Secondary ion mass spectrometry (SIMS), on the other hand, has high sensitivity and detection limits that can reach the level of parts per billion (Gannoun et al., 2011; Cerqueira et al., 2011). Unfortunately, SIMS requires high-vacuum environments that greatly restrict its ability to analyze environmental samples-especially aqueous samples. Given these considerations, laser ablation inductively coupled plasma mass spectrometry (LA-ICP-MS) appears to be a more suitable choice for the analysis of environmental samples (Wang et al., 2012) its ICP-MS component ensures high sensitivity and no organic solvent is required for the laser ablation process. The only challenge that remains for LA-ICP-MS analysis is to optimize specimen preparation.

In this paper, we report a green analytical route that combines microextraction within an inexpensive disposable pipette (Hu et al., 2010) using an ammonium pyrrolidine dithiocarbamate/methyl isobutyl ketone (APDCMIBK) system, with quantitative analysis through LA-ICP-MS (Wang et al., 2012). This approach requires only $200 \mu \mathrm{L}$ of organic solvent to complete the entire analysis process. Figure 1 outlines the concept of this proposed analytical route. We examined its validity through replicate analyses of cadmium (Cd) in NIST 1640(a) reference nature water. We also demonstrated the main advantage of this process-its simplicity with respect to sample preparation —-through analyses of Cd in samples of tap water, groundwater, and seawater.

\section{Experimental}

First, confirm all chemicals were of analytical grade and were used directly. To establish calibration curves for

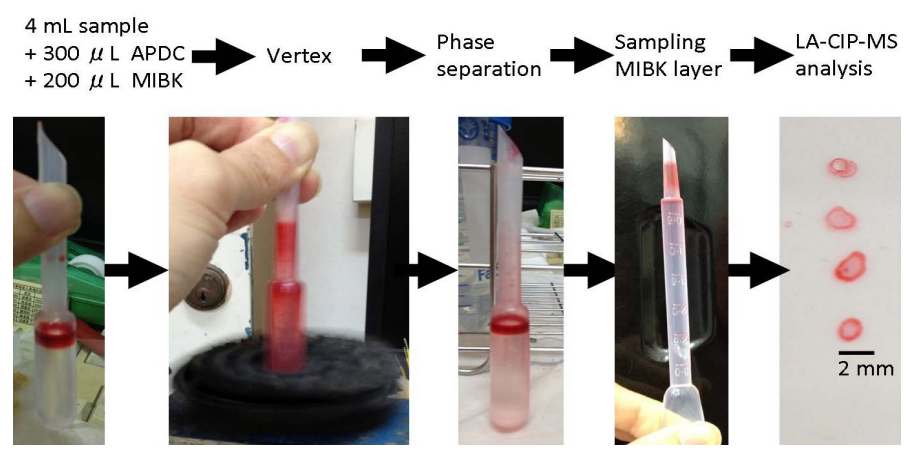

Figure 1. Schematic representation of the concept of our proposed green analytical route. 
quantification of $\mathrm{Cd}$ and $\mathrm{Pb}$, aliquots of $\mathrm{Cd}$ and $\mathrm{Pb}$ stock solutions [prepared by dissolving $\mathrm{Cd}\left(\mathrm{NO}_{3}\right)_{2}$ and $\mathrm{Pb}\left(\mathrm{NO}_{3}\right)_{2}$ in deionized water, respectively] were diluted directly with MIBK. The as-prepared standards were spiked with Oil Red O (Sigma, CAS Number 1320-06-5) to visualize their locations on the substrate and allow better focusing of the laser shot. These standards were vortexed vigorously to minimize any possible phase separation prior to placing droplets onto the substrates. The procedure for the replicate analyses of NIST 1640(a) nature water, as well as other real samples (tap water, lake water, and seawater), is outlined as follows:

1) Mix sample $(5 \mathrm{~mL})$ with APDC $(300 \mu \mathrm{L})$ in a 50 -mL polypropylene centrifuge tube, adjust the $\mathrm{pH}$ to 3 , and then vortex to induce extraction.

2) Transfer $4.5 \mathrm{~mL}$ of the mixture into a cut plastic pipette, add dye-spiked MIBK (200 $\mu \mathrm{L})$, vortex for $30 \mathrm{~s}$ to partition chelated APDC into the MIBK phase, and then set aside for phase separation.

3) Place a drop $(0.1 \mu \mathrm{L})$ of colored MIBK onto the substrate, dry, and then perform LA-ICP-MS analysis.

The following operating conditions were applied for the LA-ICP-MS system: LA was performed using a UP 213 laser ablation system (New Wave Research, USA) combined with a Nd:YAG laser (wavelength: $213 \mathrm{~nm}$ ) and operated in Q-switched (pulsed) mode. The pulse length was 4 ns with a repetition rate of $10 \mathrm{~Hz}$, a dwell time of $8 \mathrm{~s}$, and an intersite pause of $1 \mathrm{~s}$. During ablation, the laser beam (diameter: $0.11 \mathrm{~mm}$; defocused distance: $1.5 \mathrm{~mm}$ ) with fluence of $15-20 \mathrm{~J} \mathrm{~cm}^{-2}$ was used to vaporize the elements within a selected area. At a scan speed of $0.1 \mathrm{~mm} \mathrm{~s}^{-1}$ and a frequency of $10 \mathrm{~Hz}$, an area of approximately $10 \mathrm{~mm} \times 10 \mathrm{~mm}$ (depending on the actual size of the sample) was ablated and introduced into the ICP-MS system using argon as the carrier gas (1.0 L $\mathrm{min}^{-1}$ ). Analyses of the elements $\mathrm{Cd}$ and $\mathrm{Pb}$ were conducted using a quadrupole ICP mass spectrometer (Agilent 7500a, USA); the operating RF power was $1.5 \mathrm{~kW}$, and the detector was operated in time-resolved analysis acquisition mode; the plasma gas flow rate and auxiliary gas flow rate were 15 and $2.0 \mathrm{~L} \mathrm{~min}^{-1}$, respectively.

\section{Result and Discussion}

\subsection{Effect of $\mathrm{pH}$}

The APDC-MIBK extraction system has been demonstrated previously to exhibit high performance when extracting most transition metal elements from complex matrices, including seawater and industrial influent (Sun \& Sun, 2007). When co-precipitating with APDC complexes, 42 elements covering most transition metal elements (including rare earth elements) can be isolated efficiently from highly saline samples; importantly, no IA or IIA elements are extracted from these solutions at the same time (Sun \& Sun, 2007) Further pH adjustment to an appropriate working window can effectively isolate some of these 42 elements. Figure 2 displays, in good agreement with the literature, that $\mathrm{pH} 3$ is the most efficient working window for isolating the Cd element (Sun

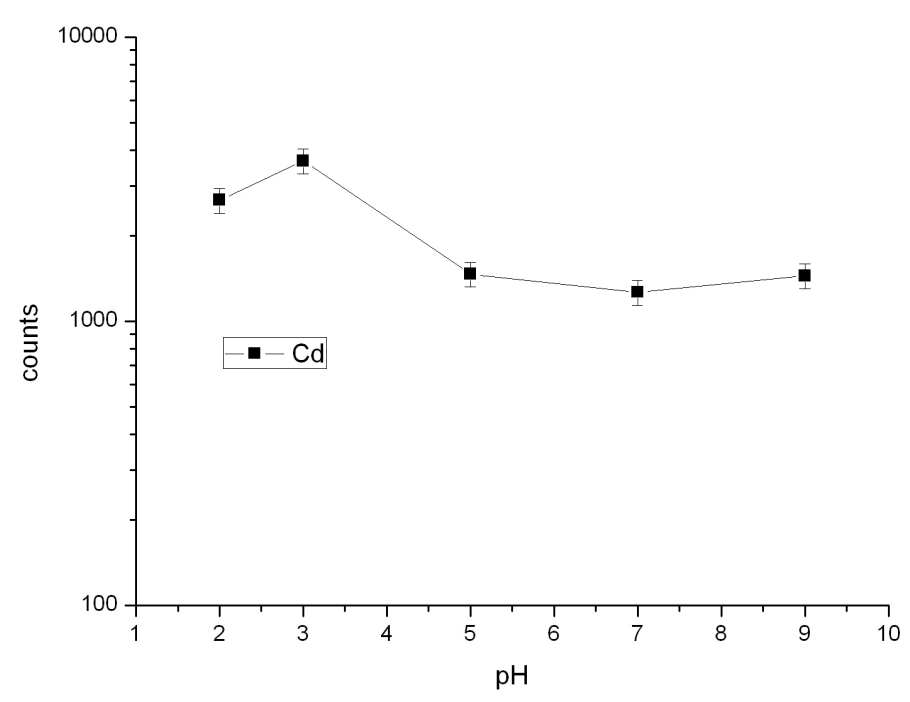

Figure 2. Intensities of signals for Cd in LA-ICP-MS analyses of samples extracted from environments at various values of $\mathrm{pH}$. The extractions were conducted using our proposed system and samples with initial Cd concentrations of 5 ppm. 
\& Sun, 2007). Accordingly, we adjusted our samples to $\mathrm{pH} 3$ in all subsequent analyses. It should be kept in mind that a further optimization can be reached by considering the adjustment in the microextraction time, salting out, complexing agent concentration, buffer concentration, organic solvent volume, sample volume, etc.

\subsection{Capability of Proposed Microextraction Route}

Having determined the working $\mathrm{pH}$ window, we required corresponding calibration curves as a prerequisite to performing further analyses. Figure 3 displays the corresponding Cd calibration curve determined using LAICP-MS. The value of $R^{2}(>0.99)$ indicates good linear responses toward $\mathrm{Cd}$ and $\mathrm{Pb}$ in the region up to $200 \mathrm{ppb}$ (ca. $1.78 \mu \mathrm{M}$ for $\mathrm{Cd}$ ). This performance suggested that the technique would be applicable for the analyses of environmental samples. We first tested the validity of our proposed microextraction procedure through analyses of NIST 1640(a), a standard reference natural water. Replicate analyses of NIST 1640(a) provided recoveries of 95 $\pm 3 \%$ for Cd (Table 1). The corresponding detection limits, derived from three standard deviations of blank analyses, of our system were $0.6 \mu \mathrm{g} \mathrm{L}{ }^{-1}$ for Cd.

Despite having observed excellent detection limits and good recoveries from replicate analyses of NIST 1640(a) nature water, the concern to analytical chemists is often the matrix effect, rather than the detection limit. That is, the superior sensitivity of ICP-MS makes it possible to determine the levels of most of the elements of any kind in an environmental sample, although the matrix of a real sample usually leads to isobaric interference as well as polyatomic interference. Minimizing these interferences is an important aspect of improving the precision of an analysis. Furthermore, clogging can occur as a result of dissolved solids depositing at the interfaces of the components-not only deteriorating the analytical performance but also greatly increase the need for maintenance. Dilution is a quite simple and straightforward approach that has been applied widely to minimize matrix effects; unfortunately, the analytes themselves will be simultaneously diluted and, in this case, worsen the detection limit of the ICP-MS system. All of these concerns inhibit the direct introduction of real samples and highlight the inevitability of sample preparation when dealing with them.

The greatest advantage that our proposed analytical method has over others is its simplicity in terms of sample preparation. Herein, we demonstrate this feature through the quantification of Cd in tap water, groundwater, and seawater (Figure 4). Considering that the concentrations of these trace samples are typically close to the detection limit of ICP-MS (Ye et al., 2012; Boda \& Sheikh 2012; Ebert \& Bhushan, 2012; Xu \& He 2012; Hsieh et al., 2011; Wang et al., 2009), we applied a standard addition method to perform replicate quantification. The details of the inorganic compositions of these real samples can be found elsewhere (Ye et al., 2012;

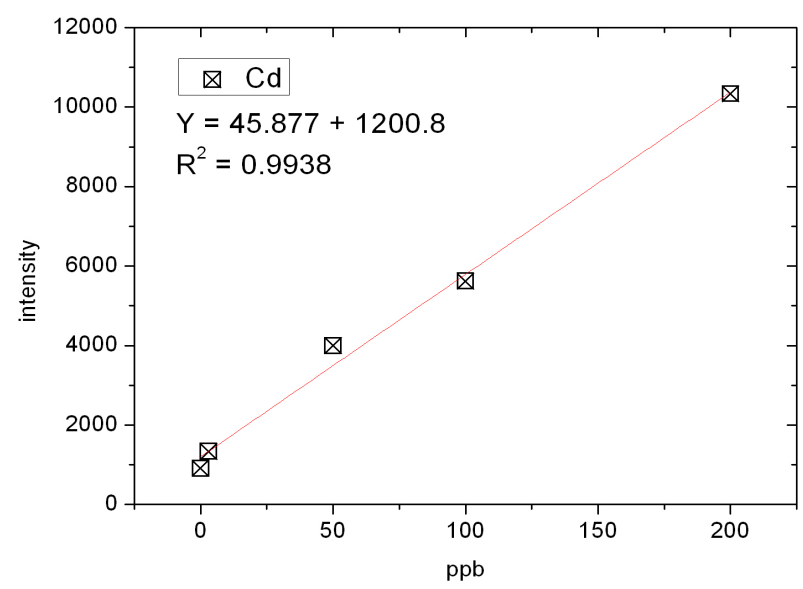

Figure 3. Calibration curves for Cd determined using LAICP-MS $(n=3)$.

Table 1. Concentrations of Cd determined through analyses of NIST 1640(a) $(n=3)$.

\begin{tabular}{ccccc}
\hline Element & Certified $(\mathrm{ppb})$ & Detected $(\mathrm{ppb})$ & Recovery (\%) & RSD (\%) \\
\hline $\mathrm{Cd}$ & $3.961 \pm 0.072$ & $3.8 \pm 0.3$ & $96 \pm 7$ & 7.89 \\
\hline
\end{tabular}




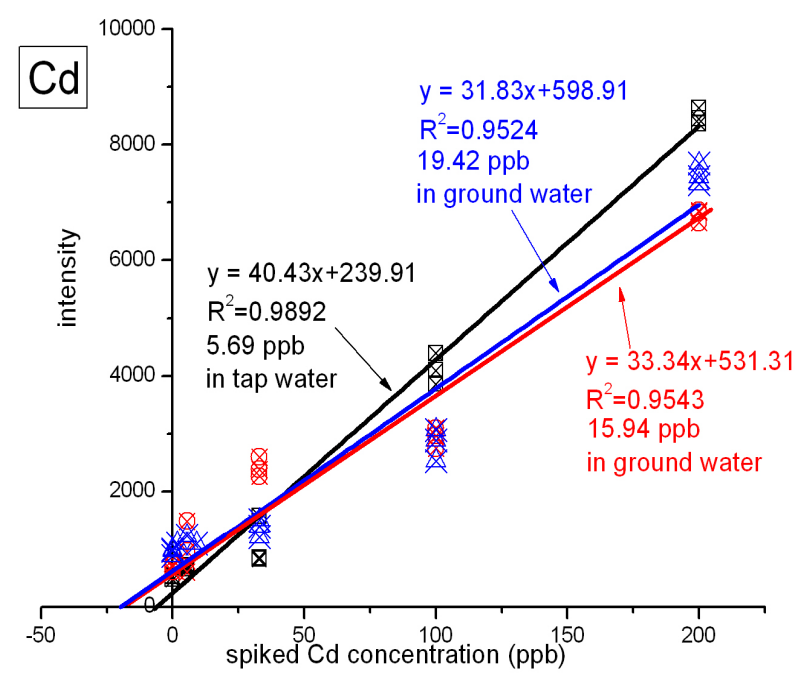

Figure 4. Analyses of real samples $(n=3)$ : tap water (black line), groundwater (blue line), and seawater (red line); calculated total dissolved solids: tap water, ca. $50 \mathrm{mg} \mathrm{L}^{-1}$; (Heish et al., 2011) groundwater, ca. 1 wt\%; (Wang et al., 2009) seawater, ca. 3.5 wt\% (Wang et al., 2010).

Boda \& Sheikh 2012; Ebert \& Bhushan, 2012; Xu \& He 2012; Hsieh et al., 2011; Wang et al., 2009), their ionic strengths range from the sub-millimolar to the tens of millimolar level (i.e., the ionic strength is proportional to the total dissolved solid content). From Figure 4, we note that the values of $R^{2}$ obtained from the tap water sample were higher than those determined from the groundwater and seawater samples. Similar to that of NIST 1640(a) nature water, the ionic strength of the tap water sample was rather low in comparison with those of the other two samples, and we suspect that this feature might have led to the better values of $R^{2}$. On the other hand, good linear responses remained in the cases of the groundwater and seawater samples. The slightly lower values of $R^{2}$ might reflect interference from the matrix in these two real samples. Nevertheless, the high extraction efficiency of the APDC-MIBK system meant that our proposed analytical method was still capable of quantifying trace amounts of $\mathrm{Cd}$ in groundwater and seawater samples. Through linear regression, we determined the concentrations of Cd to be $5.69 \mathrm{ppb}$ in tap water; $15.94 \mathrm{ppb}$ in groundwater; and 19.42 in seawater. Importantly, the simplicity, low cost, and low consumption of organic solvent make this approach a very promising green analytical method for future application to a wide variety of environmental samples.

\section{Conclusion}

In this study, we have developed a technique for green analysis through the combination of an APDC-MIBK microextraction system (in an inexpensive disposable pipette) and LA-ICP-MS. We examined the performance of this system through replicate analyses of the concentrations of $\mathrm{Cd}$ and Pb in NIST 1640(a) nature water. The entire experiment required only $200 \mu \mathrm{L}$ of organic solvent and provided an enhancement factor of 25 -a value that might improve further through judicious choice of a more-appropriate substrate. Using this system, the detection limits, determined based on three times the standard deviations, were $0.6 \mu \mathrm{g} \mathrm{L}^{-1}$ for Cd and $0.9 \mu \mathrm{g} \mathrm{L}{ }^{-1}$ for $\mathrm{Pb}$. Such sensitivities suggest that this approach would be applicable to the analyses of most environmental samples with good accuracy.

\section{Acknowledgements}

We thank the National Science Council of the Republic of China (NSC100-2221-E-008-039-MY3) for supporting this study. We appreciate Peter Glink for the detail English correction.

\section{References}

Batrusaitis, J. Chen, H. H., Rubasinghege, G., \& Grassian, V. H. (2012). Heterogeneous Atmospheric Chemistry of Lead 
Oxide Particles with Nitrogen Dioxide Increases Lead Solubility: Environmental and Health Implications. Environmental Science \& Technology, 46, 12806-12813. http://dx.doi.org/10.1021/es3019572

Beiraghi, A. Babaee, S., \& Roshdi, M. (2012). Simultaneous Preconcentration of Cadmium, Cobalt and Nickel in Water Samples by Cationic Micellar Precipitation and Their Determination by Inductively Coupled Plasma-Optical Emission Spectrometry. Microchemical Journal, 100, 66-71. http://dx.doi.org/10.1016/j.microc.2011.09.003

Bendicho, C., Lavilla, I., Pena-Pereira, F., \& Romero, V. (2012). Green Chemistry in Analytical Atomic Spectrometry: A Review. Journal of Analytical Atomic Spectrometry, 27, 1831-1857. http://dx.doi.org/10.1039/c2ja30214d

Boda, A., \& Sheikh, M. A. (2012). Density Functional Theoretical Investigation of Remarkably High Selectivity of the Cs ${ }^{+}$ Ion over the $\mathrm{Na}^{+}$Ion toward Macrocyclic Hybrid Calix-Bis-Crown Ether. The Journal of Physical Chemistry A, 116, 8615-8623. http://dx.doi.org/10.1021/jp303817s

Cerqueira, B., Vega, F. A., Serra, C., Silva, L. F. O., \& Andrade, M. L. (2011). Time of Flight Secondary Ion Mass Spectrometry and High-Resolution Transmission Electron Microscopy/Energy Dispersive Spectroscopy: A Preliminary Study of the Distribution of $\mathrm{Cu}^{2+}$ and $\mathrm{Cu}^{2+} / \mathrm{Pb}^{2+}$ on a Bt Horizon Surfaces. Journal of Hazardous Materials, 195, 422-431.

http://dx.doi.org/10.1016/j.jhazmat.2011.08.059

Ebert D., \& Bhushan, B. (2012). Durable Lotus-Effect Surfaces with Hierarchical Structure Using Micro- and Nanosized Hydrophobic Silica Particles. Journal of Colloid and Interface Science, 368, 584-591. http://dx.doi.org/10.1016/j.jcis.2011.09.049

Gannoun, A., Boyet, M., El Goresy, A., \& Devouard, B. (2011). REE and Actinide Microdistribution in Sahara 97072 and ALHA77295 EH3 Chondrites: A Combined Cosmochemical and Petrologic Investigation. Geochimica et Cosmochimica Acta, 75, 3269-3289. http://dx.doi.org/10.1016/j.gca.2011.03.017

Howard, D. L., de Jonge, M. D., Lau, D., Hay, D., Varcoe-Cocks, M., Ryan, C. G., Kirkham, R., Moorhead, G., Paterson, D., \& Thurrowgood, D. (2012). High-Definition X-Ray Fluorescence Elemental Mapping of Paintings. Analytical Chemistry, 84, 3278-3286. http://dx.doi.org/10.1021/ac203462h

Hsieh, H. F., Chen, Y. H., \& Wang, C. F. (2011). A Magnesium Hydroxide Preconcentration/Matrix Reduction Method for the Analysis of Rare Earth Elements in Water Samples Using Laser Ablation Inductively Coupled Plasma Mass Spectrometry. Talanta, 85, 983-990. http://dx.doi.org/10.1016/j.talanta.2011.05.011

Hu, X. Z., Wu, J. H., \& Feng, Y. Q. (2010). Molecular Complex-Based Dispersive Liquid-Liquid Microextraction: Analysis of Polar Compounds in Aqueous Solution. Journal of Chromatography A, 1217, 7010-7016. http://dx.doi.org/10.1016/j.chroma.2010.09.013

Iraji, A., Afzali, D., Mostafavi, A., \& Fayazi, M. (2012). Ultrasound-Assisted Emulsification Microextraction for Separation of Trace Amounts of Antimony Prior to FAAS Determination. Microchimica Acta, 176, 185-192. http://dx.doi.org/10.1007/s00604-011-0706-0

Meeravali, N. N., \& Kumar, S. J. (2012). Determination of Cd, Pb, Cu, Ni and Mn in Effluents and Natural Waters by a Novel Salt Induced Mixed-Micelle Cloud Point Extraction Using ETAAS. Analytical Methods, 4, 2435-2440. http://dx.doi.org/10.1039/c2ay25216c

Moradi, M., \& Yamini, Y. (2012). Surfactant Roles in Modern Sample Preparation Techniques: A Review. Journal of Separation Science, 35, 2319-2340. http://dx.doi.org/10.1002/jssc.201200368

Shaha, F., Kazia, T. G., Afridi, H. I., Naeemullah, A. M. B., \& Baig, J. A. (2011). Cloud Point Extraction for Determination of Lead in Blood Samples of Children, Using Different Ligands Prior to Analysis by Flame Atomic Absorption Spectrometry: A Multivariate Study. Journal of Hazardous Materials, 192, 1132-1139.

http://dx.doi.org/10.1016/j.jhazmat.2011.06.017

Sun, Y. L., \& Sun, M. (2007). Determination of 42 Trace Elements in Seawater by Inductively Coupled Plasma Mass Spectrometry after APDC Chelate Co-Precipitation Combined with Iron. Analytical Letters, 40, 2391-2404. http://dx.doi.org/10.1080/00032710701576056

Tehrani, M. S., Azar, P. A., Husain, S. W., \& Shafaei, F. (2010). Dispersive Liquid-Liquid Microextraction of Cr(VI) in Water and Hair Samples by Electrothermal Atomic Absorption Spectrometry. Asian Journal of Chemistry, 22, 6302-6310.

Teresa Pena, M., Vecino-Bello, X., Carmen Casais, M. C., Carmen Mejuto, M., \& Cela, R. (2012). Optimization of a Dispersive Liquid-Liquid Microextraction Method for the Analysis of Benzotriazoles and Benzothiazoles in Water Samples. Analytical and Bioanalytical Chemistry, 402, 1679-1695. http://dx.doi.org/10.1007/s00216-011-5598-7

Wang, T. H., Hsieh, H. A., Hsieh, Y. K., Chiang, C. S., Sun, Y. C., \& Wang, C. F. (2012). The Fate and in Vivo Biodistribution of CdSe Quantum Dots in Rat Tissues: A Laser Ablation Inductively Coupled Plasma Mass Spectrometry Study. Analytical and Bioanalytical Chemistry, 404, 3025-3036. http://dx.doi.org/10.1007/s00216-012-6417-5

Wang, T. H., Li, M. H., \& Teng, S. P. (2009). Bridging the Gap between Batch and Column Experiments: A Case Study of Cs Adsorption on Granite under High Solid/Liquid Ratio. Journal of Hazardous Materials, 161, 409-415. 
http://dx.doi.org/10.1016/j.jhazmat.2008.03.112

Wang, T. H., Li, M. H., Wei, Y. Y., \& Teng, S. P. (2010). Desorption of Cesium from Granite under Various Aqueous Conditions. Applied Radiation and Isotopes, 68, 2140-2146. http://dx.doi.org/10.1016/j.apradiso.2010.07.005

West, M., Ellis, A. T., Potts, P. J., Streli, C., Vanhoof, C., Wegrzynek, D., \& Wobrauschek, P. (2011). Atomic Spectrometry Update: X-Ray Fluorescence Spectrometry. Journal of Analytical Atomic Spectrometry, 26, 1919-1963. http://dx.doi.org/10.1039/c1ja90038b

Xu, L. G., \& He, J. H. (2012). Fabrication of Highly Transparent Superhydrophobic Coatings from Hollow Silica Nanoparticles. Langmuir, 28, 7512-7518. http://dx.doi.org/10.1021/la301420p

Ye, G., Bai, F. F., Wei, J. C., Wang, J. C., \& Chen, J. (2012). Novel Polysiloxane Resin Functionalized with Dicyclohexano-18-Crown-6 (DCH18C6): Synthesis, Characterization and Extraction of Sr(II) in High Acidity $\mathrm{HNO}_{3} \mathrm{Medium}$. Journal of Hazardous Materials, 225, 8-14. http://dx.doi.org/10.1016/j.jhazmat.2012.04.020

Zeng, C. J., Lin, Y., Zhou, N., Zheng, J. T., \& Zhang, W. (2012). Room Temperature Ionic Liquids Enhanced the Speciation of $\mathrm{Cr}(\mathrm{VI})$ and $\mathrm{Cr}(\mathrm{III})$ by Hollow Fiber Liquid Phase Microextraction Combined with Flame Atomic Absorption Spectrometry. Journal of Hazardous Materials, 237, 365-370. http://dx.doi.org/10.1016/j.jhazmat.2012.08.061 